\title{
Integration of Physics Fundamentals to Prepare Students for the Hi-Tech World through Design of Filters Deployable in Mobile Communication
}

\section{Dr. Kanti Prasad, University of Massachusetts, Lowell}

Dr. Kanti Prasad is a professor in the department of electrical and computer Engineering and is founding Director of Microelectronics/VLSI Technology Laboratories at the University Massachusetts Lowell. Professor Prasad initiated the Microelectronics/ VLSI program in 1984, and is teaching 16.469/16.502 VLSI Design and 16.470/504 VLSI Fabrication courses since its inception. From the spring of 1986 Professor Prasad developed 16.661 Local Area/Computer Networks, and since 1994 VHDL Based Digital Design and taught up to 2001, till Dr. Terence Kelly (received his doctorate under supervision of Professor Prasad) took over. From spring 1998, Professor Prasad also developed and taught 16.517, MMIC Design and Fabrication course to meet the growing demand of regional semiconductor industries. He is the recipient of Zone I best paper award by American Society of Engineering Education (ASEE) in 2008. $\mathrm{He}$ has been appointed as honorable member of IAAB of the MEGHE group of Institution and Shree Baba Ramdeo College of Engineering and Management (Nagpur) in India. He has also received the Best Teaching award for the New England Region, and the Best Campus award for the Zone 1 from ASEE during 2012. He is also coordinator for Graduate Studies in VLSI and Semiconductors certificate program. Professor Prasad already offered Online 16.517 MMIC Design and Fabrication during Spring 2009 and also developing MEMS Design and Fabrication to be offered Online starting from Spring 2013. He is the author of over 150 theses, dissertations and papers published and presented in journals/conferences of national and international repute. In 2013 Professor Prasad was awarded Fellow from the ASEE. 


\title{
Integration of Physics Fundamentals to Prepare Students for the Hi-Tech World through Design of Filters Deployable in Mobile Communication
}

\begin{abstract}
This paper brings in-depth details of piezoelectric phenomenon and its application for filters based on electromagnetic and acoustic coupling. The Silicon Oxide and Aluminum Nitride which have very low and high acoustic impedances respectively which are used in the solidly mounted resonator. This is a prime ingredient for bulk acoustic wave filters. Through integration of piezoelectricity is highlighted in the paper. It also puts physics as the most innovative tool for the Hi-Tech industries.
\end{abstract}

\section{Introduction}

Frequency selection devices such as filters can be used to manipulate different RF bands at widely varying frequencies in all mobile communication systems. Thin film Piezo-electric materials offer great opportunities that can be engineered with other materials to achieve RF Filtering. The foundation of developing experimental filters lies at acquiring a couple of stacked crystal filters (SCFs) and placing them vertically over each other separated by a thin film electrode. The SCF works on the principle of generating and transferring acoustic energy from one Piezoelectric film to another at a particular range of frequencies wherein the structure is resonant. In other words, electrical energy is converted into mechanical energy by the input Piezo-electric film and it is transferred to the output Piezo-film which converts mechanical energy back to electrical energy. The electromechanical coupling determines the amount of energy that can be converted between electromagnetic and mechanical energies within a single Piezo-electric film. In addition the amount of coupling between the two Piezo-electric films determines the mechanical energy that can be transferred between them. Generally, all aspects of the electromagnetic coupling in the device play important roles in determining the filter's resonant frequency, insertion loss and bandwidth characteristics. The approach to improve electromechanical coupling by proper choice of electrode materials, device impedance matching and beneficial use of external components like inductors are of paramount importance. The Piezoelectric films are mounted on an acoustic mirror to prevent loss of acoustic 
energy into the substrate. The acoustic mirror also serves in suppressing unwanted (spurious) modes of vibration.

Innovative mobile applications demand newer techniques, novel processes and, at the same time, should be integratable with standard CMOS Technology. Bulk Acoustic Wave (BAW) filters form core elements for highly advanced RF frontend modules. BAW filters are extremely important due to their size, low insertion loss and their integratibility with newer technologies in RF Domain. Accurate modeling of electrical performance of the RF filters depends on how precisely the values of physical parameters of materials used in their design are known and whether all of its specifications have been met. Sophisticated modeling techniques incorporating material parameters pertaining to their design, simulation and verification need to be explored to their fullest extent before fabrication of such devices. Generally, filter parameters vary from one material manufacturer to another and the processing methods used during fabrication. Therefore a methodology is needed to determine these values based on the filters electrical characteristics. In essence, a simple, expedient and inexpensive method is needed to determine effective parameters such as acoustic velocity and density values of the materials used in the filter. The innovative design of experiments (DOE) is required comprised of generating various electrical impedance peaks in the wideband spectrum of the filter that is material sensitive and correlates to the acoustic properties of the systems.

Frequency selection devices such as filters are used to manipulate different RF Bands at widely varying frequencies in all wireless communication devices. Thin film Piezoelectric Materials' offer great properties that can be engineered with other materials to achieve RF Filtering. The ElectroMechanical coupling determines the amount of energy that can be converted between a electromagnetic and mechanical within a single Piezoelectric film. Acoustic coupling between the two Piezoelectric films also determines the mechanical energy that can be transferred between them. Electromechanical coupling plays a vital role in determining the center frequency, insertion loss and bandwidth characteristics.

As the application for mobile devices continue to grow, there will be an increasing demand for the number of bands such as PCS for all phones, GPS for location, Bluetooth for short range portable devices and ISM for data. More frequencies will be crowding the spectrum. Hence filters are needed to be built such that they have high Selectivity to prevent interference from the 
adjacent bands. Band pass filters selectively transmit the signal in the pass band and reject the signals in the stop band as shown in Fig 1.

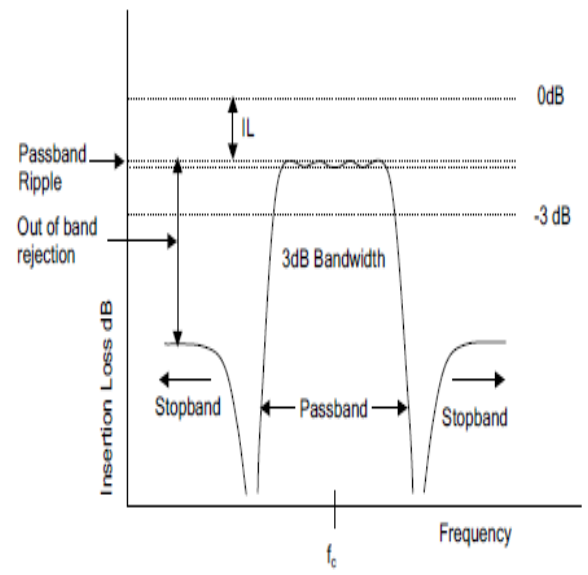

Figure 1

Insertion Loss (IL) is the attenuation of the signal on the pass band caused by insertion of the filter. Low values of IL are desired for low power to enhance the battery life. Half of the power is lost in a filter with an IL of $3 \mathrm{~dB}$. Low values of IL are desired for better sensitivity and quality of the signal.

\section{Bulk Acoustic Wave (BAW) Technology}

The technology provides promising solutions to meet the growing demand. In Bulk mode acoustic filters, energy is stored as both electromagnetic and mechanical energy through piezoelectric coupling. A schematic of thin film BAW resonator is shown in Fig 2.

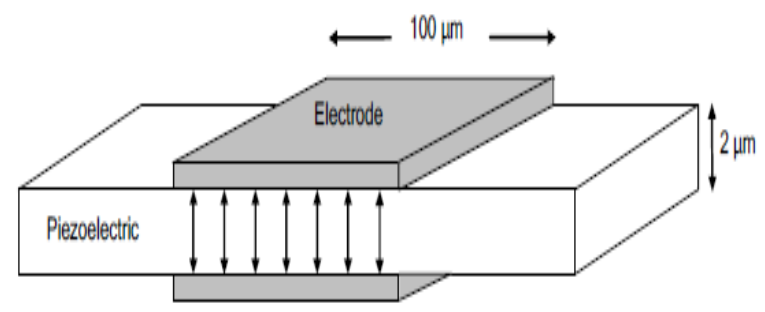

Figure 2 
The thickness of the film is approximately equal to half the wavelength required by dielectric resonator. Since the velocity of sound in Piezo electric layer is much smaller than that of light, the devices are 3-4 orders of magnitude smaller than dielectric resonation. Thickness control of the Piezoelectric film becomes extremely important to achieve the desired center frequency.

\section{Solidly Mounted Resonator (SMR)}

The thin film BAW resonator can be mounted as shown in Fig 3.
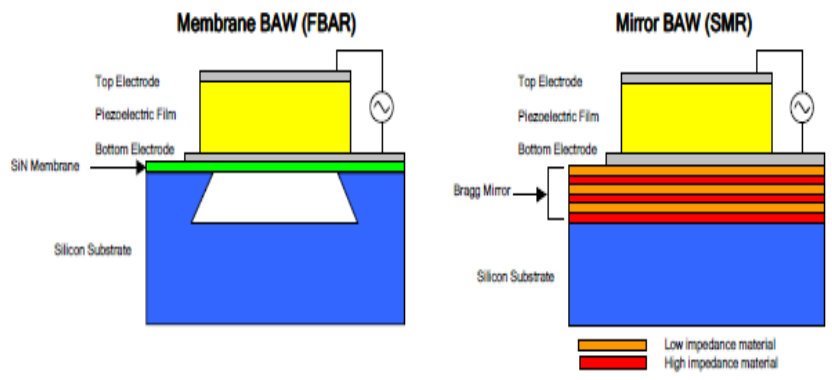

Figure 3

On an acoustic mirror, also known as Bragg's reflector. In SMR, the Bragg mirror is made up of layers of alternate low and high impedances of thickness roughly equal to quarter wavelength of the desired center frequency. This analogous to transmission line impedance transformer. Bragg mirror generally uses materials such as Tungsten (W) and Silicon Oxide (SiO2). SMR's are rugged and easy to process. They meet most of the desired needs.

In Stacked Crystal Resonator filters, the two resonators are directly placed over each other whereas in coupled resonator filters the resonators are separated by a layered structure so that coupling between base resonator can be controlled. The construction of a stacked crystal filter is as shown in Fig 4. 


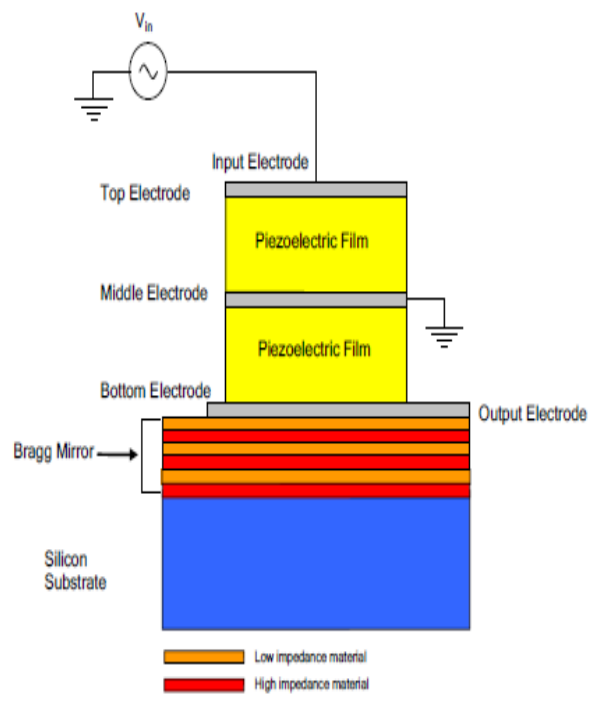

Figure 4

Although the electrical signal is applied to the top resonator, the capacitor of the bottom resonator forms a permanent structure, thereby determining the resonance frequency. The top resonator generates mechanical vibrations and converts it back into electrical signals. The acoustic coupling between the resonators determines the bandwidth and size of the filter. The acoustic coupling is the maximum with Piezoelectric films of equal thickness. In this case the first mode is half wavelength across the whole structure, that is, there is a quarter wavelength across the whole structure. In the second mode, there are wavelengths across the whole structure, that is, half wavelength across each structure. The schematic of a coupled resonator filter is as shown in Fig 5

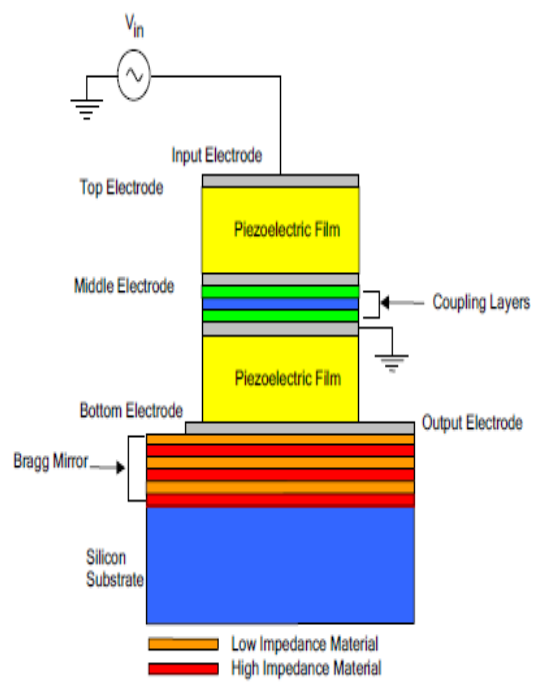

Figure 5 
The coupling layers control the acoustic coupling between two resonators. The choice of materials for coupling layers determines achievable bandwidth, performance and tunability.

\section{Piezoelectric Effect}

Piezooelectric materials such as Barium Titanate ( BaTiO3) Aluminium Nitrate (AIN), Lead Zirconate Titanate (PZT), have built in electrical dipoles in their crystal lattice. These electrical dipoles generate mechanical stress and elongation in presence of an electrical field. The material can contract or expand depending on the direction of Electromagnetic field as shown in Fig 6.

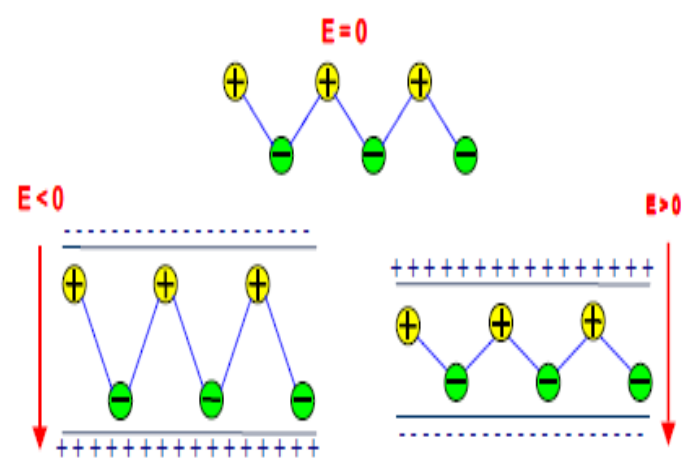

Figure 6

If Piezoelectric material is deformed it will generate electric field and charge.

\section{Acoustic Waves}

Particles in a solid contains many atoms, when particles are displaced, it gives rise to internal resting force, which lead to oscillating motion of the medium, giving rise to longitudinal or transverse mode. In the longitudinal mode, the motion of particles in the acoustic medium only in the direction of propagations shown in the Fig 7, where material is expanding and contracting in the direction of propagation of the acoustic wave. 


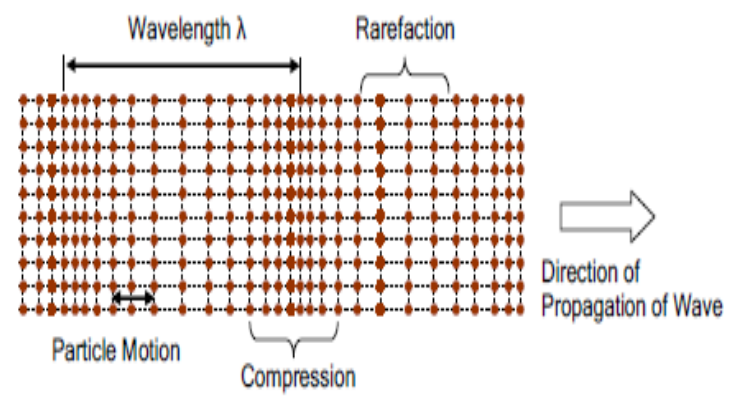

Figure 7

The longitudinal mode refers to a charge in local volume or mass density of the material. In the shear mode as shown in Fig 8, the motion is transverse and flexing or bending of the material about an axis perpendicular to the direction of propagation.

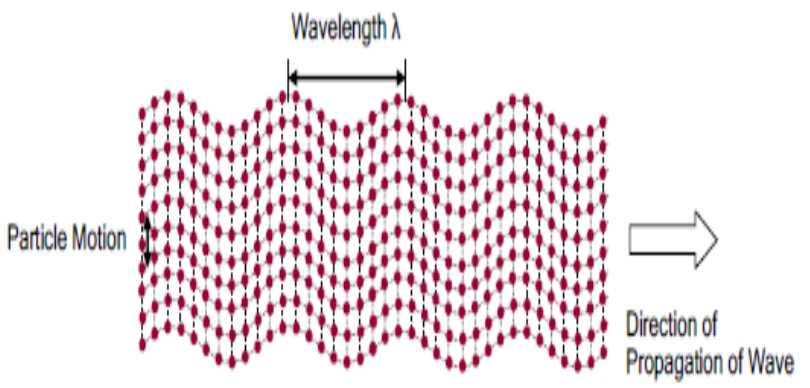

Figure 8

Even in the case of propagation medium being isotropic, the longitudinal and transverse velocities $\mathrm{V}_{\mathrm{L}}$ and $\mathrm{V}_{\mathrm{T}}$ are different

$\mathrm{V}_{\mathrm{L}}=\sqrt{\frac{C 11}{p}}$
$\mathrm{~V}_{\mathrm{T}}=\sqrt{\frac{C 44}{p}}$

Where $\mathrm{C}_{\mathrm{ij}}$ is the stiffness constant and $\mathrm{p}$ is the density of solid.

The longitudinal wave causes a volume change with propagation, whereas transverse wave does not. 
The energy associated with the vibration of the atom about the rest position in lattice is called the Phonon. This energy is proportional to the frequencies of allowed atomic vibrations. The one dimensional wave equation for acoustic waves in solids is given by

$\frac{\partial^{2} T}{\rho \partial Z^{2}}=\frac{\partial^{2} T}{c \partial t 2}$

where $\mathrm{T}$ is the stress and $\mathrm{Z}$ is the direction of propagation of the wave. Acoustic waves are extremely slow as compared to electromagnetic waves. As a consequence the wavelength of acoustic wave is very short. At $1 \mathrm{GHz}$, the wavelength is approximately 5um, which is extremely useful for miniaturization of BAW's.

Piezo Butterworth Van Dyke (BVD) Model

The model of electromagnetic behavior of the piezo material is depicted in Fig 9.

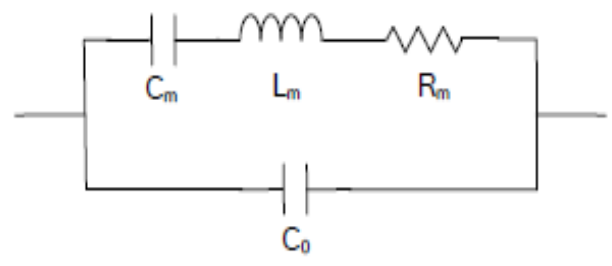

Figure 9

The BVD circuit describes the operation of a resonating structure near resonance in terms of electrical lumped elements. The BAW resonator is a capacitor at most of the frequencies and becomes an inductor in a narrowband near acoustic resonance. The area of the resonator electrodes determines the capacitor $\mathrm{Co}$, the thickness of the Piezoelectric film determines the frequency expressed in BVD as LC resonance, where

$\mathrm{C}_{\mathrm{m}}=4 \mathrm{Co} \mathrm{K}_{\mathrm{t}}^{2} / \pi^{2}$

$\mathrm{L}_{\mathrm{m}}=1 / \omega^{2} \mathrm{C}_{\mathrm{M}}$

$\mathrm{R}_{\mathrm{m}}=\omega \mathrm{L}_{\mathrm{m}} / \mathrm{Q}$

Where $\mathrm{C}_{\mathrm{m}}$, is the motional Capacitance that represents the elasticity of the resonator, $\mathrm{Lm}$ is the motional Inductance that represents the mass, $\mathrm{R}_{\mathrm{m}}$ is the acoustic energy loss and Co is the clamped capacitance between the two 
electrodes of the resonator. The electro mechanical coupling coefficient (Kt) and quality factor $(\mathrm{Q})$, can be given as

$\mathrm{K}_{\mathrm{t}}=\left(\pi^{2} / 4\right)\left(\left(\mathrm{f}_{\mathrm{P}}-\mathrm{f}_{\mathrm{S}}\right) / \mathrm{f}_{\mathrm{P}}\right)$

$\mathrm{Q}=2 \pi \mathrm{f}_{\mathrm{S}} \mathrm{L} / \mathrm{R}$

where $\mathrm{fs}$ is the resonance frequency and fp is the anti resonance frequency. The impedance response of the BVD model is depicted as given in Fig 10

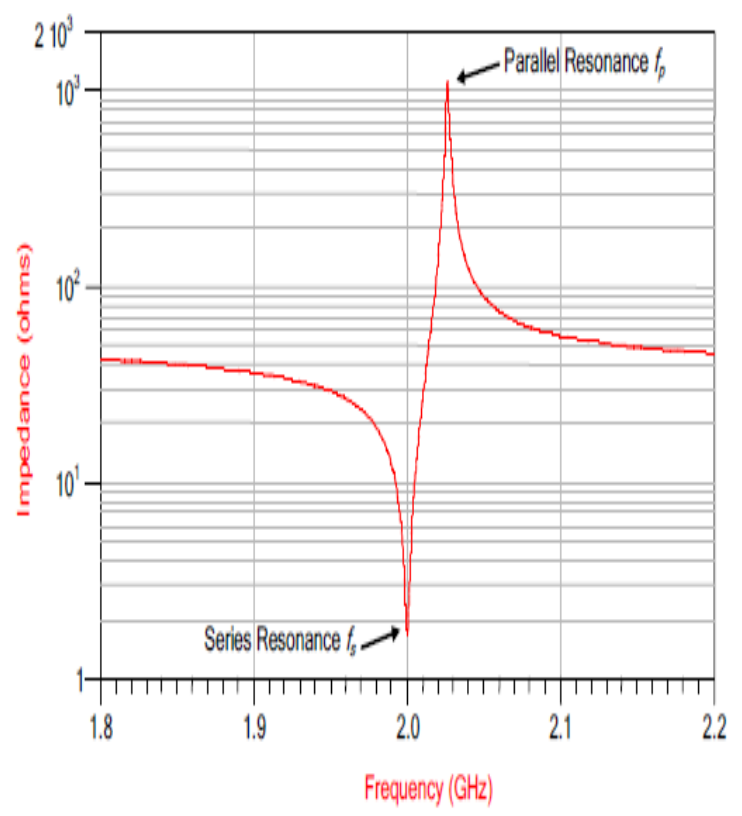

Figure 10

The resonators maybe connected in such a way that the series resonant frequency of the series resonator coincides roughly with parallel resonant frequency of the shunt resonator as shown in Fig 11. 


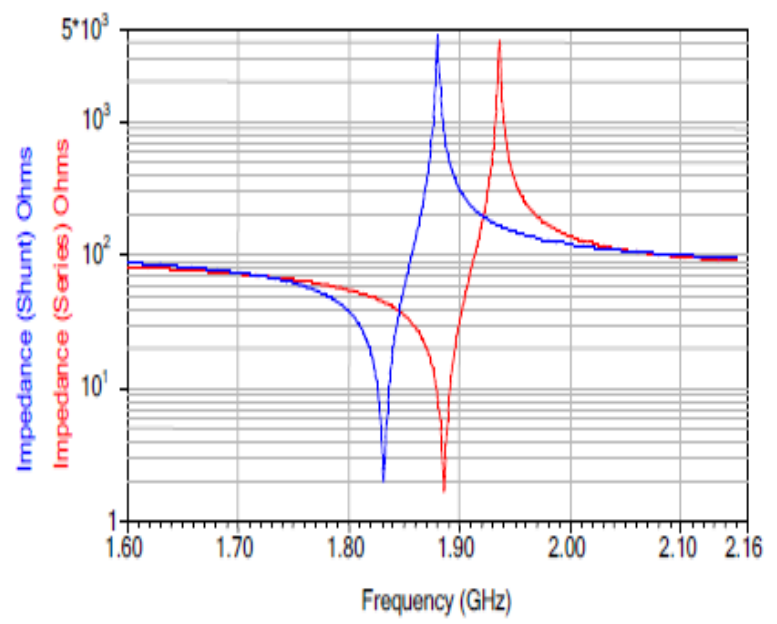

Figure 11

This frequency is called the center frequency of the filter. At this frequency there is low impedance path between input and output and a high impedance to shunt the path. Both series and shunt resonators can be fabricated on the same wafer. Fig 12 depicts the response of such a filter.

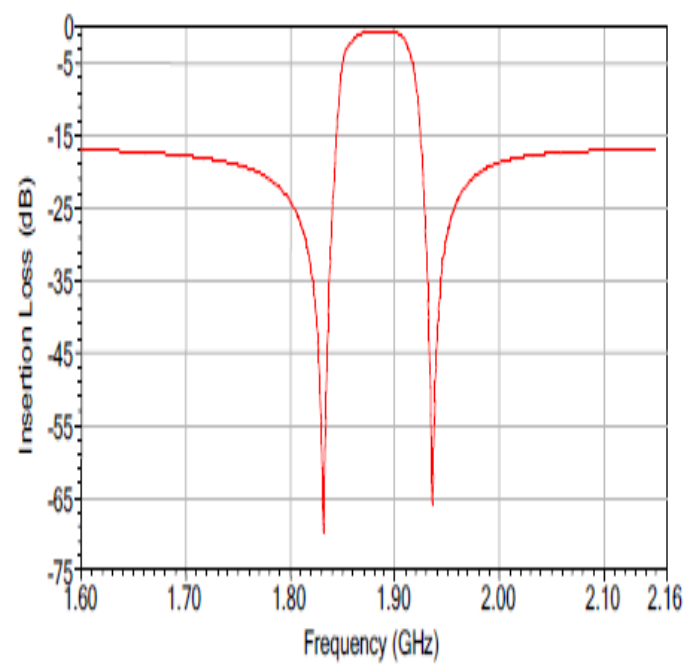

Figure 12 


\section{Conclusion}

In essence these Piezoelectric materials lead to formation of resonators which lead to Band Pass Filters in general and to deploy them in Mobile Communication in particular. I am convinced however, that innovations in Engineering Education must be carried out in all disciplines of engineering through integration of fundamentals along with the State-ofArt technologies for the readiness of the work force development nationally as well as internationally to meet the challenges of emerging technologies of the 21 st century.

The author feels a compelling need for integration of Physics fundamentals in all Hi-Tech courses. Such a methodology adequately prepares students for a demanding industrial world wherein technology is evolving all the time but basic fundamentals hardly change. Those who are successful in integrating these fundamentals with $\mathrm{Hi}$-Tech courses never face obsolescence. The author has been involved in this art of integration for the last three decades and has witnessed several hundreds of his students achieved high success in their careers. The mantra in the classroom has been only those students who successfully integrate fundamentals in Hi-Tech courses become wise, else they remain otherwise. My earnest attempt has been to place all of my students into the category of 'wise'.

\section{Acknowledgement}

I wish to thank my administration as well as the administration of regional $\mathrm{Hi}$-tech industries for supporting me in these educational endeavors. I want to especially thank the industries who have made a long term commitment for Instructional Processing and Research Investigations with the author. Thanks, are also due to my beloved wife for supporting me all along. I also want to thank my blessed Assistant for typing the manuscript. 


\section{References}

1. Kanti Prasad., "Innovations in Engineering Education through Integration of Physics" Presented at 2016 ASEE Annual Conference and Exposition in June 26-29, New Orleans.

2. Sagar Karalkar, Kanti Prasad ,Yu Zhu, Jerod Mason, and Dylan Bartle, "Multi-Device Optimization for Scalable DC HEMT Model with Self-Heating Effect" ICESC, Nagpur India 2014.

3. Prasad K., "21st Centaury Challenges: Integrating Fundamentals Into State-Of-The-Art Technology Curricula Complimented by Hands on Experience in Laboratories" Swarthmore College, PA, Nov, 2014.

4. Prasad K., "Advanced VLSI Training Being Imparted Regionally, Nationally and Internationally ", ASEE's North East Conference held at Norwich University at Vermont (March 15-16th, 2013).

5. Prasad K., Invited Presentation "Mixing Fundamentals with State -ofArt Technology for preparing the students for 21st century" at BRCEM Nagpur, India Jan 2012.

6. Prasad K., "Preparing the students for 21 st century with a proper mix of Fundamentals with state-of-Art Technology in Collaboration with regional Hi-Tech Industry" , ICC April 2012

7. Ambarish Roy, Bradley P. Barber, and Kanti Prasad, "Modeling of RF-MEMS BAW Resonator," International Conference on VLSI Design, Bangalore India, 2010.

8. Ambarish Roy, Bradley P. Barber, Vinay Kulkarni and Kanti Prasad, "Spurious Mode Suppression in Stacked Crystal Filters," IEEE Long Island Systems, Applications and Technology Conference, Farmingdale, NY, 2010.

9. Ambarish Roy, Bradley P. Barber, and Kanti Prasad, "Thin Film Solidly Mounted Resonators for RF Devices," $12^{\text {th }}$ International Symposium on Microwave and Optical Technology, New Delhi, India 2009.

10. Ambarish Roy, Bradley P. Barber, Vinay S. Kulkarni, and Kanti Prasad, "Material Acoustic Speed and Density Parameter Extraction in Solidly Mounted Resonators," student paper in IEEE Sarnoff Symposium, Princeton, NJ, 2009.

11. Ambarish Roy, Bradley P. Barber, and Kanti Prasad, "Microwave Filter and Dedicated Sensor-an Integrated Resonator," student paper in IEEE Sarnoff Symposium, Princeton, NJ, 2010. 